\title{
NOTE ON THE PRECEDING PAPER
}

\section{IRVING KAPLANSKY AND HARRY POLLARD}

The sufficiency portion of the theorem on the harmonic series proved by Erdös and Niven in the preceding paper hinges on the fact that (in their notation) $k_{2}=k$ implies $k_{j}=k$ for $j>2$. We shall show that this is true more generally for any series $\sum u_{n}$ such that $\left\{u_{n}\right\}$ is completely monotonic. The result follows at once from the theorem below.

In the case $k_{2}>k$, the method has thus far not yielded any result of the kind obtained by Erdös and Niven.

THEOREM. Let $u_{n} \neq 0(n=1,2, \cdots)$ be a sequence such that

$$
(-1)^{k} \Delta^{k} u_{n} \geqq 0 \quad(k=0,1, \cdots ; n=1,2, \cdots),
$$

that is, $\left\{u_{n}\right\}$ is completely monotonic, and

Define

$$
\lim _{n \rightarrow \infty} u_{n+1} / u_{n}=1 \text {. }
$$

$$
\begin{aligned}
& S(n, k)=u_{n}+u_{n+1}+\cdots+u_{n+k-1}, \\
& f(n, k)=S(n+k, k+1)-S(n, k) .
\end{aligned}
$$

Then $f(n, k)>0$ implies $f(n+1, k)>0$.

We require the following lemma, which is a consequence of a theorem of D. V. Widder. ${ }^{1}$

LEMMA. Let $\phi(t)$ be a function continuous in $(0,1)$ and having at most one change of sign in this interval. If $\alpha(t)$ is non-decreasing in $(0,1)$, then the sequence $v_{n}$ defined by

$$
v_{n}=\int_{0}^{1} t^{n} \phi(t) d \alpha(t), \quad n=1,2, \cdots,
$$

has at most one change of sign.

Proof. If $\phi(t)$ is of constant sign in $(0,1)$ there is nothing to prove. Suppose then that it changes sign at $t=t_{0}$. Define $\psi(t)=\int_{t_{0}}^{t} \phi(t) d \alpha(t)$. Then $\psi(t)$ has at most one change of $\operatorname{trend}^{2}$ in $(0,1)$. Since

Received by the editors November 26, 1944.

1 D. V. Widder, The inversion of the Laplace integral and the related moment problem, Trans. Amer. Math. Soc. vol. 36 (1934) p. 195.

${ }^{2}$ Loc. cit. p. 155. 
$v_{n}=\int_{0}^{1} t^{n} d \psi(t)$, the result follows from the theorem of Widder. ${ }^{1}$

PROOF OF THE THEOREM. A well known result of Hausdorff states that a sequence satisfying (1) admits the representation

$$
u_{n}=\int_{0}^{1} t^{n} d \alpha(t)
$$

where $\alpha(t)$ is non-decreasing. ${ }^{3}$ It follows that

$$
f(n, k)=\int_{0}^{1} t^{n} \phi(t) d \alpha(t)
$$

where $\phi(t)=\left(t^{2 k+1}-2 t^{k}+1\right) /(t-1)$. It is readily verified that $\phi(t)$ has exactly one change of sign in $(0,1)$; hence the same is true for the function $\phi(t)-\epsilon$, for sufficiently small $\epsilon>0$, say $\epsilon<\epsilon_{0}$. Thus by the lemma the sequence

$$
f(n, k)-\epsilon u_{n}=\int_{0}^{1} t^{n}[\phi(t)-\epsilon] d \alpha(t)
$$

has at most one change of sign for $\epsilon<\epsilon_{0}$.

Suppose now that $f(n, k)>0$, while $f(n+1, k) \leqq 0$. From (2) it follows that $f(N, k)>0$ for a large enough $N>n+1$. Choose $\epsilon_{1}, 0<\epsilon_{1}<\epsilon_{0}$, so small that

$$
\begin{aligned}
f(n, k)-\epsilon_{1} u_{n} & >0, \\
f(n+1, k)-\epsilon_{1} u_{n+1} & <0, \\
f(N, k)-\epsilon_{1} u_{N} & >0 .
\end{aligned}
$$

Then the sequence $f(n, k)-\epsilon_{1} u_{n}$ has at least two changes of sign. But this contradicts the remark above that it can have at most one change of sign. This completes the proof.

New York City

${ }^{3}$ Loc. cit. p. 191. 